\title{
Laminar Transformation of Frequency Organization in Auditory Cortex
}

\author{
Daniel E. Winkowski ${ }^{1}$ and Patrick 0. Kanold ${ }^{1,2}$ \\ ${ }^{1}$ Institute for Systems Research and ${ }^{2}$ Department of Biology, University of Maryland, College Park, Maryland 20742
}

The mammalian neocortex is a six-layered structure organized into radial columns. Within sensory cortical areas, information enters in the thalamorecipient layer and is further processed in supragranular and infragranular layers. Within the neocortex, topographic maps of stimulus features are present, but whether topographic patterns of active neurons change between laminae is unknown. Here, we used in vivo two-photon $\mathrm{Ca}^{2+}$ imaging to probe the organization of the mouse primary auditory cortex and show that the spatial organization of neural response properties (frequency tuning) within the thalamorecipient layer (L3b/4) is more homogeneous than in supragranular layers (L2/3). Moreover, stimulus-related correlations between pairs of neurons are higher in the thalamorecipient layer, whereas stimulus-independent trial-to-trial covariance is higher in supragranular neurons. These findings reveal a transformation of sensory representations that occurs between layers within the auditory cortex, which could generate sequentially more complex analysis of the acoustic scene incorporating a broad range of spectrotemporal sound features.

\section{Introduction}

Sensory information from the periphery arrives in the cortex via thalamocortical afferents that terminate in the middle (thalamorecipient) cortical layers. After entering into the cortex, sensory information is further processed within columns spanning multiple cortical laminae (Atencio et al., 2009; Sakata and Harris, 2009; Sharpee et al., 2011). While prior studies have characterized topographic maps of stimulus features (Hubel and Wiesel, 1977; Mountcastle, 1997; Schreiner and Winer, 2007) across the cortical surface or the differences in stimulus selectivity of individual neurons between the cortical laminae (Armstrong-James et al., 1992; Brumberg et al., 1999; Atencio and Schreiner, 2010), the spatial transformation of feature representation within populations of neurons across cortical laminae is unknown. Here, we investigate the transformation of the representation of sound frequency in mouse primary auditory cortex (A1).

Neurons in the auditory system respond best to sounds in a narrow range of frequencies. Tonotopy, the spatially ordered gradient of neuronal frequency preference, originates in the cochlea and is preserved through all stages of the auditory system to varying degrees. Tonotopic gradients have been reported in A1 of many mammalian species including human, primate, and cat (Kaas, 2011) (but see Abeles and Goldstein, 1970). In mouse

Received June 30, 2012; revised Nov. 16, 2012; accepted Nov. 23, 2012

Author contributions: D.E.W. and P.O.K. designed research; D.E.W. performed research; D.E.W. analyzed data; D.E.W. and P.O.K. wrote the paper.

This work was supported by NIDCD Grants R01DC009607 (P.0.K.) and R01DC005779 (Shihab A. Shamma). We are grateful to Dr. Xiangying Meng for advice on statistical analyses. We thank Drs. Shihab A. Shamma and D. Butts and P. Watkins for helpful discussions and. S. Shamma, P. Manis, A. Reyes, D. Butts, S. David, B. Englitz, P. Watkins, A. Jones, K. Orzechowski, and A. Sheikh for comments on earlier versions of this manuscript.

The authors declare no competing financial interests.

Correspondence should be addressed to Patrick 0. Kanold, Department of Biology, University of Maryland, 1116 Biosciences Research Building, College Park, MD 20742. E-mail: pkanold@umd.edu.

DOI:10.1523/JNEUROSCI.3101-12.2013

Copyright $\odot 2013$ the authors $\quad 0270-6474 / 13 / 331498-11 \$ 15.00 / 0$ models, though, the existence of precise tonotopic gradients appears somewhat unresolved. For example, multiunit electrophysiological studies in the thalamorecipient layer (L3b/4) of anesthetized mouse have described smooth, precise tonotopic gradients in A1 (Stiebler et al., 1997; Hackett et al., 2011; Guo et al., 2012), whereas in vivo 2-photon $\mathrm{Ca}^{2+}$ imaging studies of populations of single neurons in the supragranular layer (L2/3) of anesthetized mouse show fractured tonotopic gradients in $\mathrm{A} 1$ on fine spatial scales while still maintaining tonotopic gradients on larger spatial scales (Bandyopadhyay et al., 2010; Rothschild et al., 2010). While some differences between these studies could be partially explained by fundamental differences in the experimental approach (electrophysiology vs $\mathrm{Ca}^{2+}$ imaging), the higher heterogeneity observed in supragranular layer with imaging approaches could point to specific transformations occurring between these cortical laminae (i.e., L4 and L2/3).

Since $\mathrm{L} 2 / 3$ neurons receive inputs not only from thalamorecipient neurons (L4) but also significant inputs from other L2/3 neurons, we speculated that there might be differences in the functional organization of neurons within L4 and L2/3, and that such differences could begin to elucidate the nature of the transformation of sensory representations within local cortical circuitry and resolve the apparent contradictions of prior studies.

\section{Materials and Methods}

Animal preparation. In vivo two-photon imaging was performed as described previously (Bandyopadhyay et al., 2010). Mice of either sex [postnatal day 24 (P24)-P43; C57/BL6; Jackson Laboratories] were prepared for acute experiments by inducing surgical levels of anesthesia with 2-3\% isoflurane in $100 \%$ oxygen. A plate for securing the head was attached to the skull and a $2 \mathrm{~mm}$ diameter craniotomy was performed above the auditory cortex. Throughout all procedures, the animal's skin temperature was maintained at $33-35^{\circ} \mathrm{C}$ (which corresponds to a core temperature of $37^{\circ} \mathrm{C}$, data not shown) with a heating plate. The ear canal contralateral to the imaging location was accessed by making an incision from the ventral side of the ear canal. All procedures were approved by 
A

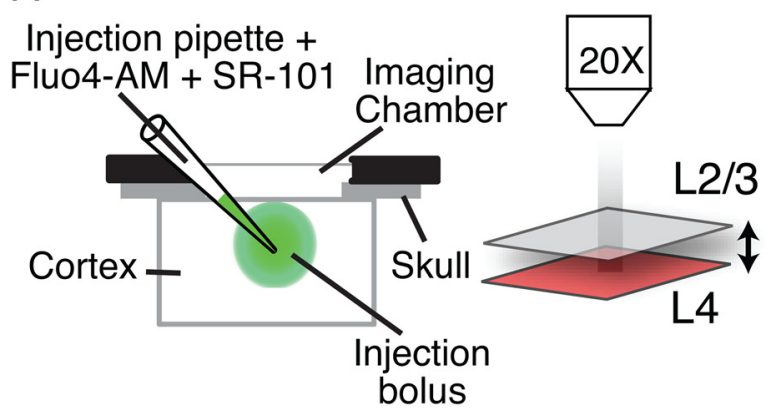

C

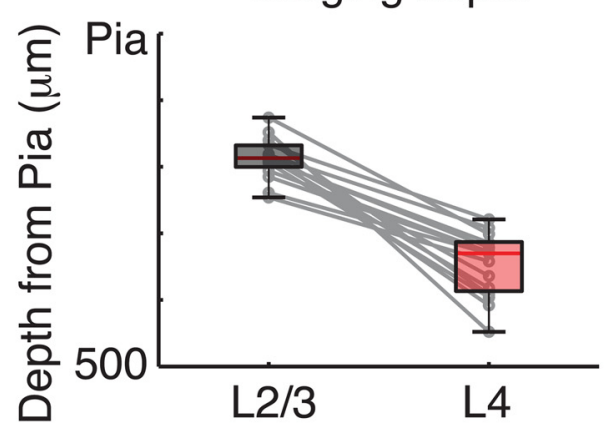

D

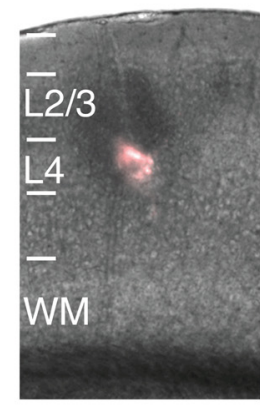

Figure 1. Imaging in multiple lamina in auditory cortex. $A$. Schematic of experimental setup. $\boldsymbol{B}$, Image of loaded neurons in L4. Scale bar, $50 \mu \mathrm{m}$. C, Mean imaging depths for the two groups (L2/3 mean depth from pia, $190 \pm 32 \mu \mathrm{m} ; \mathrm{L} 4$ mean depth from pia, $348 \pm 46 \mu \mathrm{m}$; average separation of imaging planes, $161 \mu \mathrm{m})$. $\boldsymbol{D}$, Left, Injections of latex microspheres at L4 imaging depth confirm imaging in midcortical layers. Right, Expression of the layer 4-specific marker Scnn1a (Madisen et al., 2010; Viswanathan et al., 2012) indicates location of $L 4$. We crossed a transgenic mouse expressing cre recombinase under the control of Scnn1a promoter [B6;C3-Tg(Scnn1a-cre)3Aibs/J; JAX strain 9613], with a mouse that expresses td Tomato in a cre-dependent manner [B6.Cg-Gt(ROSA)26Sortm9(CAG-tdTomato)Hze; Jax strain 7909]. WM, White matter.

the University of Maryland Institutional Animal Care and Use Committee.

In vivo two-photon imaging. Dye solution (containing the $\mathrm{Ca}^{2+}$ indicator dye Fluo-4 AM) (Bandyopadhyay et al., 2010) was pressure injected into Al with a pipette $\sim 400-500 \mu \mathrm{m}$ from the cortical surface. Sulforhodamine-101 was included in the pipette solution to visualize astrocytes (Nimmerjahn et al., 2004). Following the completion of the loading protocol, isoflurane anesthesia was reduced to $0.5-0.75 \%$. After loading of neurons was achieved ( $\sim 60 \mathrm{~min}$ after injection), the craniotomy was covered with warm agarose (1-1.5\%) and coverslipped to create a stable imaging window. Imaging of sound-evoked $\mathrm{Ca}^{2+}$ transients was performed using a commercially available two-photon microscope (Prairie Technologies) controlled by PrairieView software. Excitation light from a MaiTai DeepSee laser (Newport) with dispersion compensation was scanned by galvanometers through a $20 \times 0.95$ NA objective (Olympus). For imaging Fluo-4 AM, the laser was tuned to $800 \mathrm{~nm}$. For each sound stimulus, a sequence of $20-25$ full-frame images $(256 \times 256$ pixels) was acquired at $\sim 7 \mathrm{~Hz}$ (144 ms frame period, $6-8 \mu$ s dwell time) with stimulus onset occurring at frame number 7 . Overall baseline luminance within each pair of imaging fields (i.e., L2/3 and L4) was maintained by adjusting the incident laser power while keeping the photomultiplier tube gain constant.

Sound stimuli. Sound stimuli (13 or 17 sinusoidally amplitudemodulated tones; $6-48$ or $4-64 \mathrm{kHz} ; 0.25$ octave spacing; $\sim 60 \mathrm{~dB}$ SPL; $1 \mathrm{~s}$ long; $5 \mathrm{~Hz}$ full-depth modulation; 6-7 s interstimulus interval; $10 \mathrm{~ms}$ linear on and off ramps) were generated and attenuated using a TuckerDavis Technologies RX6 and PA5. We used calibrated TDT EC1 speakers (driven with TDT ED1 drivers) and delivered sounds through a coupled tube positioned close to the ear canal. The sound system was calibrated between 6 and $70 \mathrm{kHz}$ and showed a flat spectrum over this range. Overall SPL at $0 \mathrm{~dB}$ attenuation was $\sim 80 \mathrm{~dB}$ SPL on average (for tones). Each stimulus was repeated 10 times.
L4-RFP

Image analysis. Imaging analysis was performed as described previously (Bandyopadhyay et al., 2010). Data were collected and analyzed from 16 paired fields of view (i.e., L2/3 and L4) from 11 mice. For each imaging field, individual neurons were identified manually from the average of all collected images. Fluorescence of neurons was measured in circular regions (three to five pixel radius; $3.5-6 \mu \mathrm{m}$ ) over the cell soma. The change in fluorescence $(\Delta F)$ relative to the baseline fluorescence $(F)$ over time was determined for each repeat and then averaged. The stimulus was on for the first six frames after stimulus onset (1 s). The $\Delta F / F$ values for individual neurons over these frames $(\sim 1$ s) for different frequencies are used for all analyses. Unless noted otherwise, estimates of error are represented as SEM. Neurons in which the calcium waveform was significantly modulated by sound presentation were defined by ANOVA $(p<0.01)$ across baseline (prestimulus) and all sound presentation periods. Frequency-tuning curves were obtained by calculating the mean response $(\Delta F / F)$ during the stimulus period for each sound frequency. Best frequency (BF) was defined as the peak (maximum $\Delta F / F$ ) of the frequency-tuning curve. To assess BF variability, we used two strategies. For assessing BF variability across entire imaging field, the interquartile range $\left(\mathrm{IQR}_{\mathrm{BF}}\right)$, in octaves, was calculated for all responding neurons within the field of view. For assessing local $(<100$ $\mu \mathrm{m}) \mathrm{BF}$ variability for each neuron, we calculated the $\mathrm{IQR}_{\mathrm{BF}}$ (in octaves) for all responding neurons within a $100 \mu \mathrm{m}$ radius around each neuron.

To classify single neurons in our population as having single-peaked or multipeaked tuning curves, we used two different criteria, described below. Neurons whose frequency-tuning curve contained a single local maximum exceeding either 60 or $75 \%$ of the maximum response were classified as single-peaked neurons; neurons whose frequency-tuning curve contained more than a single local maximum were classified as multipeaked neurons. Results with either criterion (60 or $75 \%$ ) were similar, and thus we report data from only one criterion (75\%) and note in the text that the results using the other criterion were similar.

To assess tuning curve bandwidth (BW), we first performed linear interpolation of the frequency-tuning curve to find the minimum and maximum sound frequencies $\left(F_{\min }\right.$ and $F_{\max }$, respectively) that evoked responses exceeding either 60 or $75 \%$ of the maximum $\Delta F / F$ response for each responding neuron. For single-peaked neurons, the $\log _{2}$ of the ratio $\left(F_{\max } / F_{\min }\right)$ was used as a measure of tuning bandwidth in octaves; for multipeaked neurons, the same calculation was performed as a measure of total bandwidth despite the possible absence of responses (or response below the threshold criterion) to some of the intermediate sound frequencies.

Correlations in response variability [noise correlations (NCs)] and stimulus-related cross-correlations [signal correlations (SCs)] in groups of neurons can have profound effects on population coding of sensory inputs and, thus, the functional organization of neural circuits (Averbeck et al., 2006; Josić et al., 2009; Cohen and Kohn, 2011). Signal correlation between the responses of two neurons was determined as follows. We computed the cross-correlation between two input vectors at zero lag using the Matlab function xcorr. The input vector for each neuron was the mean response $\left(\mathrm{Ca}^{2+}\right.$ waveform) over time for each sound frequency. The time-varying sequences were normalized such that their autocorrelations at zero lag were identically 1.0. The normalized crosscorrelations at zero lag between the time-varying mean responses (i.e., six 
frames after stimulus onset) of pairs of neurons to each sound stimulus were computed yielding a cross-correlation value at zero lag for each sound stimulus ( 13 or 17 for each neuron pair). The average cross-correlation value across all stimuli was considered the signal correlation value for that pair of neurons. To create a shuffled version of the data, we randomized the responses of each neuron over frequency and time and then computed signal and noise correlation for pairs of neurons in the same manner as above.

Pairwise signal correlations were also computed using the tuning curves of neurons (i.e., $\mathrm{Ca}^{2+}$ waveforms averaged during the six frames after stimulus onset for each sound frequency). The primary difference between the two methods (i.e., using the time-fluctuating responses of the neuron pair and using the frequency-tuning curves of the pair) was that the distributions of correlation values obtained with tuning curves tended to be high with a long tail extending toward 0 , indicating that, as expected, many neurons in an imaging field had roughly similar frequency preferences (or $\mathrm{BFs}$ ) at the single sound level that was used here. Here, we report on the correlations between the time-varying responses of two neurons but note that roughly similar patterns were observed between laminae when calculating signal correlations using frequency-tuning curves.

Previous studies have noted that noise correlations can significantly bias the signal correlations (Rothschild et al., 2010). Therefore, we followed the correction procedure as described previously to account for this potential confound and compared our analyses:

$$
\begin{gathered}
R\left(x\left(t_{j}\right), y\left(t_{j}\right)\right)=\frac{\operatorname{cov}(x, y)}{\sqrt{\operatorname{cov}(x, x) \times \operatorname{cov}(y, y)}}, \\
\operatorname{cov}(x, y)=\frac{1}{M} \sum_{j=1}^{M}\left(x\left(t_{j}\right)-\bar{x}\right)\left(y\left(t_{j}\right)-\bar{y}\right),
\end{gathered}
$$

where $x\left(t_{j}\right)$ and $y\left(t_{j}\right)$ correspond to the mean responses of neurons $x$ and $y$, respectively, to all repeats $\left[r_{i}^{x}\left(t_{j}\right)\right.$ and $r_{i}^{y}\left(t_{j}\right)$ with $i=1, \ldots, N$ and $t_{j}$ corresponding to the time points of the response] of a particular sound frequency (Eq. 3-4). $\bar{x}$ and $\bar{y}$ correspond to the mean of $x(t)$ and $y(t)$ over the entire time period, respectively. We have the following:

$$
\begin{aligned}
& x\left(t_{j}\right)=\frac{1}{N} \sum_{i}^{N} r_{i}^{x}\left(t_{j}\right), \\
& y\left(t_{j}\right)=\frac{1}{N} \sum_{t}^{N} r_{i}^{y}\left(t_{j}\right),
\end{aligned}
$$

The above equations become

$$
\begin{aligned}
& \left(x\left(t_{j}\right)-\bar{x}\right)=\left(\frac{1}{N} \sum_{i}^{N} r_{i}^{x}\left(t_{j}\right)-\frac{1}{M} \sum_{j}^{M} \frac{1}{N} \sum_{i}^{N} r_{i}^{x}\left(t_{j}\right)\right), \\
& \left(y\left(t_{j}\right)-\bar{y}\right)=\left(\frac{1}{N} \sum_{i}^{N} r_{i}^{y}\left(t_{j}\right)-\frac{1}{M} \sum_{j}^{M} \frac{1}{N} \sum_{i}^{N} r_{i}^{y}\left(t_{j}\right)\right),
\end{aligned}
$$

$$
\begin{aligned}
\left(x\left(t_{j}\right)-\bar{x}\right)\left(y\left(t_{j}\right)-\bar{y}\right)=\frac{1}{N^{2}}\left(\sum _ { i } \sum _ { i ^ { \prime } } \left(r_{i}^{x}\left(t_{j}\right)\right.\right. & \left.-\bar{r}^{x}\left(t_{j}\right)\right)\left(r_{i^{\prime}}^{y}\left(t_{j}\right)\right. \\
& \left.\left.-\bar{r}^{y}\left(t_{j}\right)\right)\right) .
\end{aligned}
$$

When $i=i^{\prime}$, the values resulting from this product include the contribution of noise correlation to signal correlation:

$$
\begin{array}{r}
\operatorname{cov}_{\text {corrected }}(x, y)=\frac{1}{M} \sum_{j=1}^{M}\left(\frac { 1 } { N ^ { 2 } - N } \left(\sum_{i} \sum_{i^{\prime}}\left(r_{i}^{x}\left(t_{j}\right)-\bar{r}^{x}\left(t_{j}\right)\right)\right.\right. \\
\left.\left.\cdot\left(r_{i^{\prime}}^{y}\left(t_{j}\right)-\bar{r}^{y}\left(t_{j}\right)\right)-\sum_{i^{\prime \prime}}\left(r_{i^{\prime \prime}}^{x}\left(t_{j}\right)-\bar{r}^{x}\left(t_{j}\right)\right)\left(r_{i^{\prime \prime}}^{y}\left(t_{j}\right)-\bar{r}^{y}\left(t_{j}\right)\right)\right)\right) .
\end{array}
$$

Thus, we removed these terms from the sum (Eq. 8). The correlation coefficient then becomes the following:

$$
R_{\text {corrected }}\left(x\left(t_{j}\right), y\left(t_{j}\right)\right)=\frac{\operatorname{cov}_{\text {corrected }}(x, y)}{\sqrt{\operatorname{cov}_{\text {corrected }}(x, x) \times \operatorname{cov}_{\text {corrected }}(y, y)}} .
$$

Similarly, noise correlations were computed with the Matlab function xcov at zero lag and normalized by using "coeff" as the normalization parameter. To create a shuffled version of the data, we randomized the responses of each neuron over frequency and time and then computed signal and noise correlation for pairs of neurons in the same manner as above.

Statistical tests for normality were performed using the lilliefors function in Matlab. The outcome of this test determined whether parametric or nonparametric tests were use to compare the two distributions/populations. 
A

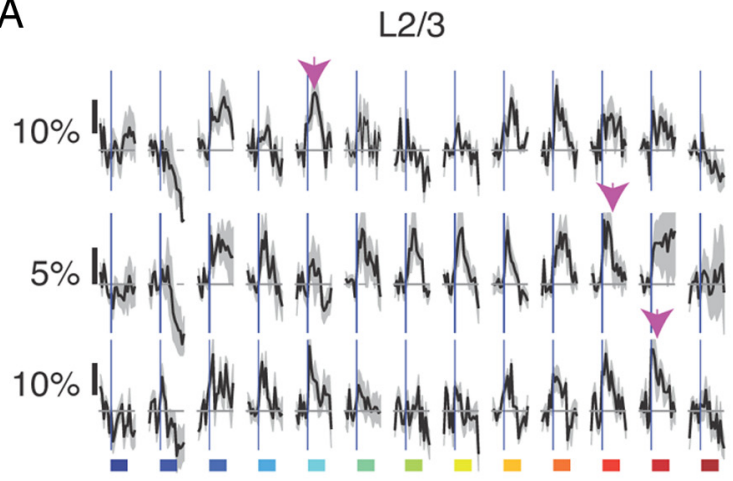

D

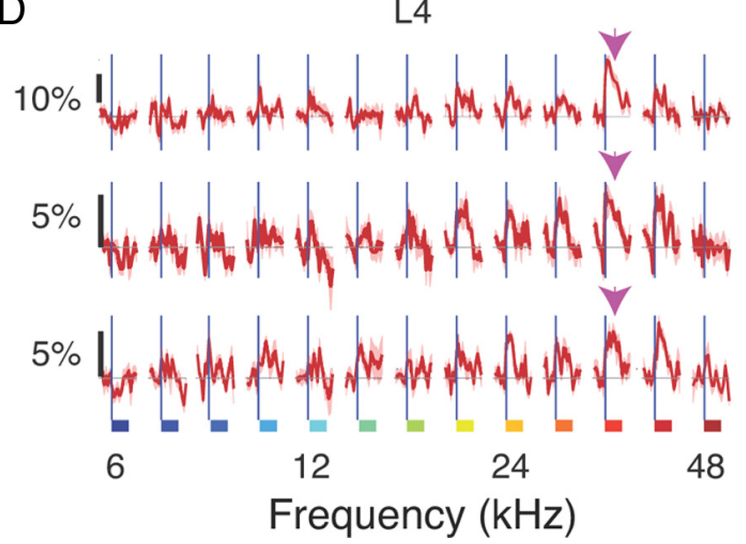

B

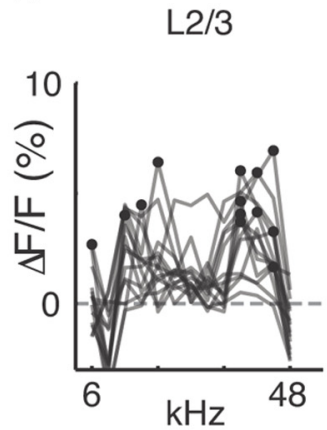

E

L4

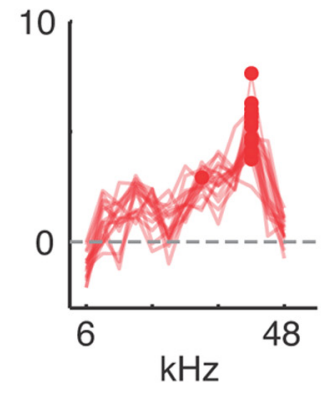

C

$\mathrm{L} 2 / 3$

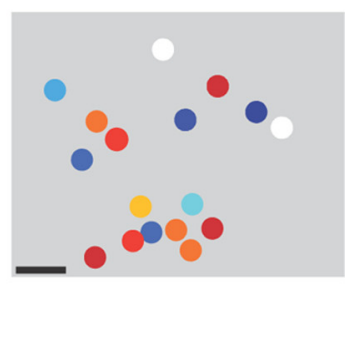

F

L4

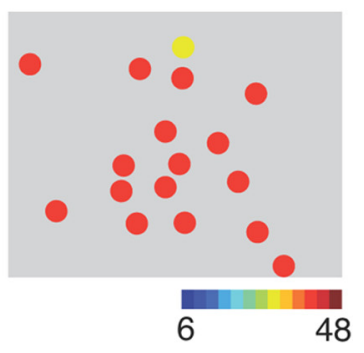

Figure 3. BF variability in $L 4$ and $L 2 / 3$. $A$, Fluorescence $(\Delta F / F)$ time course traces from three neighboring neurons in $L 2 / 3$ (rows). Horizontal colored bars indicate sound frequency and duration (1 s). Arrowheads indicate BFs. B, Superimposed tuning curves of all responsive neurons in the imaged field. Filled circles indicate the BF for each neuron. C, Spatial map of BFs for all imaged neurons in the field. Color indicates BFs. White circles indicate unresponsive neurons (see Materials and Methods). $\boldsymbol{D}-\boldsymbol{F}$, Conventions are as in $\boldsymbol{A}-\boldsymbol{C}$. $\boldsymbol{D}$, Fluorescent time course traces $(\Delta F / F)$ from three neighboring neurons in $\mathrm{L} 4$ (red). $\boldsymbol{E}$, Frequency-tuning curves of all responsive neurons in the imaged field. $\boldsymbol{F}$, Spatial map of BFs for all imaged neurons. Scale bar: $\boldsymbol{C}$ (for $\boldsymbol{C}, \boldsymbol{F}) 25 \mu \mathrm{m}$.

A

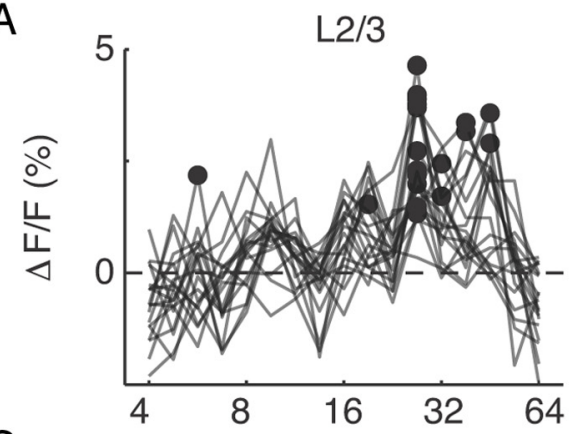

C

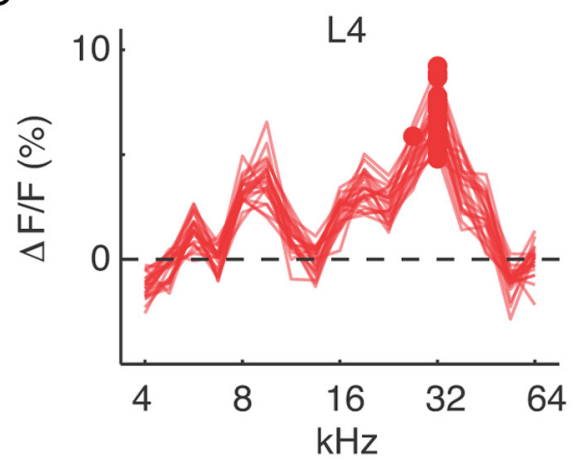

B

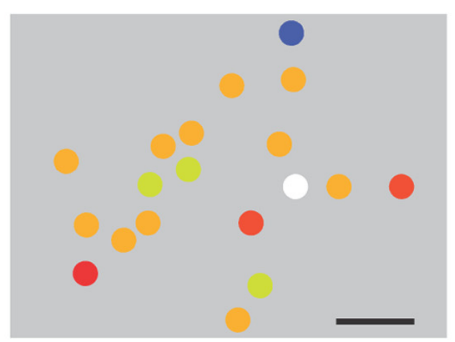

L4

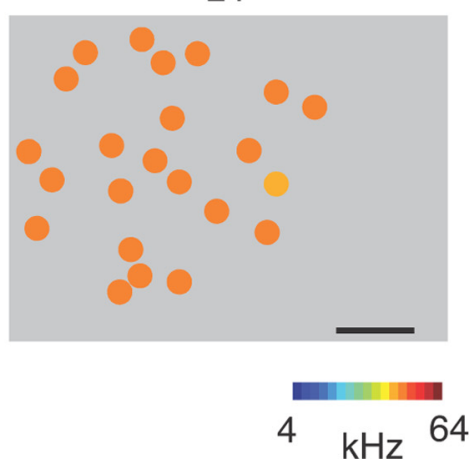

Figure 4. BF variability in $L 4$ and $L 2 / 3$. A second example of paired imaging fields. Conventions are as in Figure 3. $A$, Superimposed tuning curves of all responsive neurons in L2/3. B, Spatial map of BFs for imaged neurons in the field. $\boldsymbol{C}, \boldsymbol{D}$, Frequency-tuning and spatial maps of BF for the corresponding $L 4$ imaging field. Scale bars: $25 \mu \mathrm{m}$.

\section{Results}

In vivo imaging of L4 neurons

To investigate the functional organization of $\mathrm{A} 1$ in the laminar domain, we performed in vivo two-photon $\mathrm{Ca}^{2+}$ imaging in A1 of isoflurane-anesthetized mice (Bandyopadhyay et al., 2010). A volume of cortex was bulk loaded with the $\mathrm{Ca}^{2+}$. sensitive dye (Fluo-4 AM), and populations of neurons within the loaded region were subsequently imaged at two distinct optical planes corresponding to the L2/3 and L3b/4 (L4) by moving the objective vertically ( $z$ direction) while keeping the $x-y$ position relatively constant (Fig. $1 A-$ $C)$. Occasionally, small adjustments were made in $x$ and $y$ dimensions to maximize the number of visible dye-loaded neurons in each field (mean absolute shifts, $x=21 \pm 27 \mu \mathrm{m} ; y=20 \pm 34 \mu \mathrm{m})$. In a subset of experiments, we confirmed our imaging location and laminar position with small volume dye injections (Fig. 1D). Overall, 358 neurons were imaged at depths corresponding to L2/3, and 388 neurons were imaged at depths corresponding to $\mathrm{L} 4$ in the mouse $(n=16$ paired fields of view from 11 animals). 
L4 neurons respond more strongly to tones than $\mathrm{L} 2 / 3$ neurons

At each imaging location, to characterize the response properties of populations of identified neurons, we presented sinusoidally amplitude-modulated tones of varying frequency and monitored sound-evoked $\mathrm{Ca}^{2+}$ transients for each imaged neuron. Many imaged cells in both imaging planes showed statistically significant $\mathrm{Ca}^{2+}$ transients $(p<0.01$, ANOVA; see Materials and Methods) in response to sound stimulation $(\Delta F / F, \mathrm{~L} 2 / 3,310$ of $358 ; \mathrm{L} 4,341$ of $388)$ that were maximal at one particular frequency (Fig. 2A). To explore differences in response strength (as measured by calcium imaging techniques) among neurons in distinct imaging planes, we computed the average maximum response strength (maximum $\Delta F / F \%$ ) of all responding neurons in each imaged field and compared these values across pairs of imaging planes. We found the average response strength was significantly higher in L4 imaging fields compared with L2/3 imaging fields (mean L4, $6.46 \pm 0.91 \%$ SEM; mean L2/3, $3.96 \pm 0.56 \%$ SEM; $p<0.03$, paired $t$ test; Fig. $2 B$ ). To test whether this difference was also present when comparing the total population of neurons, we compared the strength (maximum $\Delta F / F \%$ ) of the responses in all responsive neurons within each layer. Consistent with the effects observed in pairs of fields, this analysis showed that auditory stimuli on average produced larger fluorescence changes in L4 than in L2/3 (median maximum $\Delta F / F_{\mathrm{L} 4}, 6 \%$; median maximum $\Delta F / F_{\mathrm{L} 2 / 3}, 3 \%$; Wilcoxon rank sum, $p<10^{-23}$; Fig. 2C). Since changes in fluorescence of Fluo-4 AM provide an estimate of changes in $\mathrm{Ca}^{2+}$ influx into neurons associated with spiking activity in mouse A1 (Bandyopadhyay et al., 2010; Rothschild et al., 2010), these results suggest that while the fraction of responding neurons were similar in both layers $(\sim 87 \%$ in both layers; $p>0.1$, Wilcoxon rank sum), L4 neurons might be more robustly driven by tonal sound stimuli.

\section{Frequency organization is more heterogeneous in $\mathrm{L} 2 / 3$ than L4}

We next compared the frequency preference of groups of neurons within in each layer. For each neuron, we defined its BF as the frequency producing the maximum $\Delta F / F$ value of frequencytuning curve. In the pairs of imaged fields shown in Figures 3 and 4, L2/3 neurons could show widely varying BFs similar to previous reports (Bandyopadhyay et al., 2010; Rothschild et al., 2010), indicating a large spatial variability of BF (Figs. $3 A-C, 4 A, B$ ). In contrast, L4 neurons showed significantly less $B F$ variability (Figs. 3D-F, 4C,D).

To quantitatively compare BF variability in pairs of imaging fields, we calculated the $\mathrm{IQR}_{\mathrm{BF}}$ for all responding neurons in the imaging field and compared these values across pairs of imaged fields (Fig. 5A). The $\mathrm{IQR}_{\mathrm{BF}}$ was significantly higher in $\mathrm{L} 2 / 3$ imaging fields compared with L4 imaging fields (median L2/3, $0.47 \pm$ 1.3 IQR octaves; median L4, $0.16 \pm 0.6 \mathrm{IQR}$ octaves; $p<0.01$, Wilcoxon sign rank test; Fig. $5 A$ ). In addition, we explored the local $\mathrm{IQR}_{\mathrm{BF}}$ for each neuron by calculating the $\mathrm{IQR}_{\mathrm{BF}}$ for all neurons that were located within $100 \mu \mathrm{m}$ of the central neuron. Consistent with the differences across paired imaging planes, across the population of all neurons, the local $(<100 \mu \mathrm{m}) \mathrm{IQR}_{\mathrm{BF}}$ in $\mathrm{L} 2 / 3$ was higher than the local $\mathrm{IQR}_{\mathrm{BF}}$ in $\mathrm{L} 4$ (median $\mathrm{L} 2 / 3$,

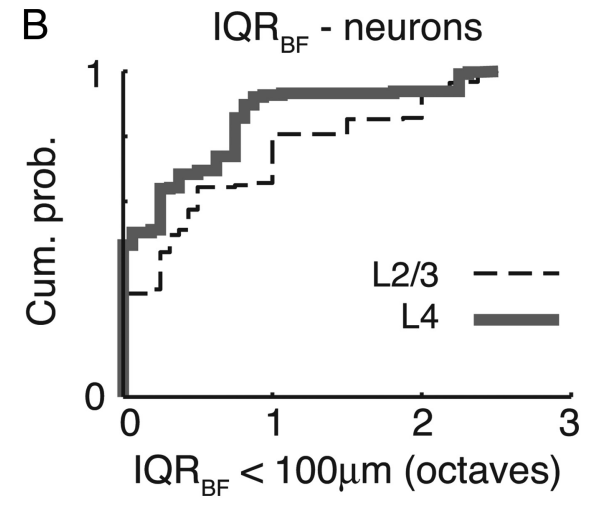

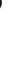

Figure 5. BF variability is larger in $L 2 / 3 . A, B F$ variability $\left(I Q R_{B F}\right)$ in each paired imaging field. Each set of connected dots represents a pair of imaging fields. $L 4$ fields showed lower variability (median ${ }_{\mathrm{L} 2 / 3}, 0.47 \pm 1.3$ octaves; $_{\text {median }}{ }_{\mathrm{L} 4}, 0.16 \pm 0.56$ octaves; $p=0.01$, Wilcoxon sign rank). $\boldsymbol{B}$, Cumulative distribution functions displaying local ( $<100 \mu \mathrm{m}$ radius) BFvariability around each neuron in L2/3 (black dashed $0.38 \pm 1$ IQR octaves; median L4, $0.06 \pm 0.75$ IQR octaves; $p<$ $10^{-6}$, Wilcoxon rank sum; Fig. $5 B$ ).

The higher BF heterogeneity of L2/3 imaging planes could be due in part to noisier measurements of tuning in L2/3 neurons (Figs. 3, 4), thus making BF determination potentially difficult. If this were the case, one might expect to find discrepancies between median BF values of the neurons in the two imaging planes. However, we found extremely good agreement between median BF values across layers in the 16 paired imaging fields $(p>0.3$, sign rank test; Fig. $6 A$ ). This suggests that our estimation of $B F$ in $L 2 / 3$ does not contribute to the larger amount of BF heterogeneity we observed in paired imaging fields, and that, in general, large scale tonotopic organization is preserved across cortical laminae. Alternatively, higher BF heterogeneity of L2/3 imaging fields could be due the presence of neurons with multipeaked tuning curves in our data and, specifically, more of them or wider peak spacing in L2/3. However, we found roughly equal fractions of neurons with single-peaked tuning curves in both laminae $(p>0.3$, paired $t$ test; Fig. $6 B$ ). This was also the case if the threshold was lowered to $60 \%$ rather than $75 \%$ of max response $(p>0.2$, paired $t$ test; data not shown). Given that similar proportions of both single-peaked and multipeaked neurons exist in mouse A1, we further analyzed two basic response characteristics (i.e., tuning bandwidth and BF variability in only neurons with single-peaked tuning) for A1 neurons and compared across L2/3 and L4. To assess tuning curve BW in only single-peaked neurons, we computed the bandwidth of the tuning curve $\left[\log _{2}\left(F_{\max } / F_{\min }\right)\right]$ that evoked responses exceeding $75 \%$ of the maximum $\Delta F / F$ response $\left(\mathrm{BW}_{75}\right)$ for each neuron and then compared these values across layers. Across the population of single-peak neurons, we found consistently larger $\mathrm{BW}_{75}$ values in $\mathrm{L} 4$ compared to $\mathrm{L} 2 / 3$ (L2/3, $0.23 \pm 0.18$ IQR octaves; L4, $0.28 \pm 0.18$ IQR octaves; $p<0.001$, Wilcoxon rank sum; Fig. $6 C$ ). This outcome was similar if the threshold applied was $60 \%$ of the maximum response for each neuron (L2/3, $0.4 \pm 0.3$ IQR octaves; L4, $0.43 \pm 0.3$ IQR octaves; $p>0.03$, Wilcoxon rank sum; data not shown). In contrast, for multipeaked neurons, there was no difference across layers in total response bandwidth at $75 \%$ of maximum $\Delta F / F$ response ( $p>0.5$, Wilcoxon rank sum; data not shown) or $60 \%$ of maximum response ( $p>0.4$, Wilcoxon rank sum; data not shown). To assess BF heterogeneity in only single-peaked neurons in paired imaging fields, we computed the $\mathrm{IQR}_{\mathrm{BF}}$ for neurons in which tuning curves contained a single peak exceeding $75 \%$ of the maximum response. We found that single-peak L2/3 neurons 
A

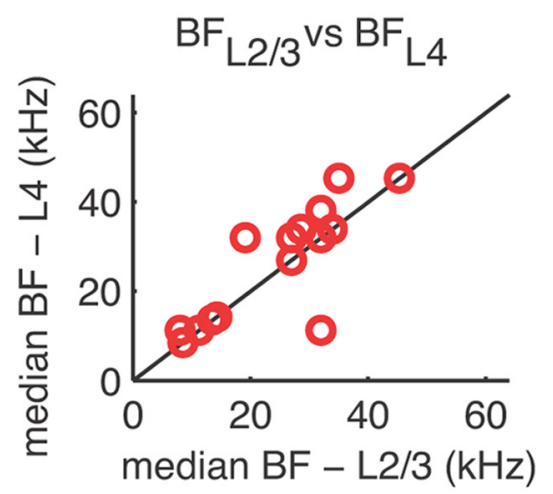

C

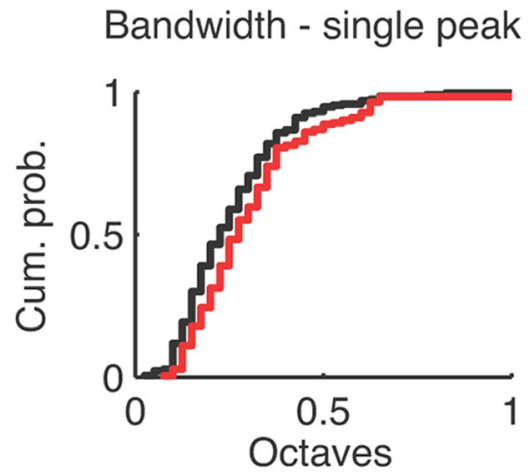

E

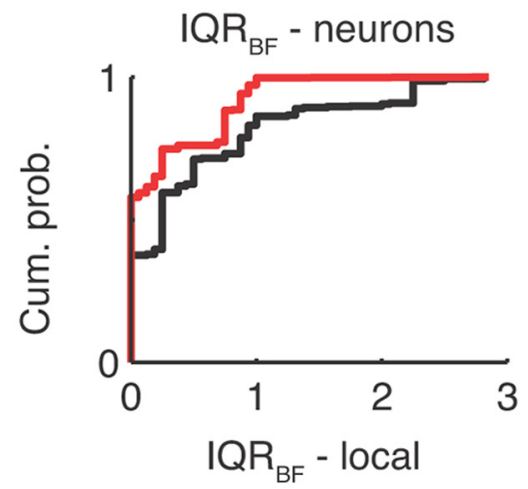

B

$\%$ single peak

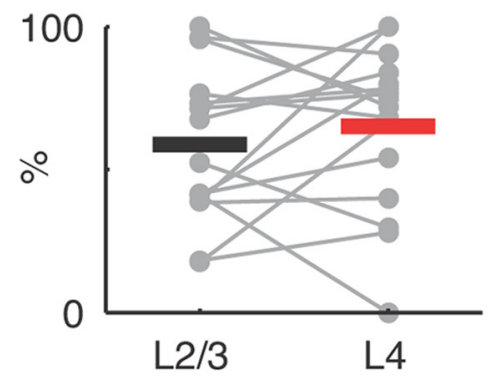

D

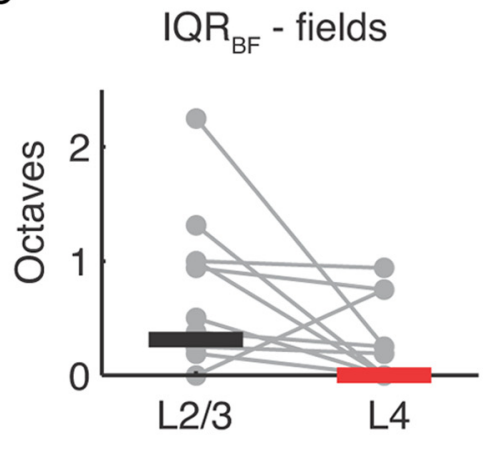

F

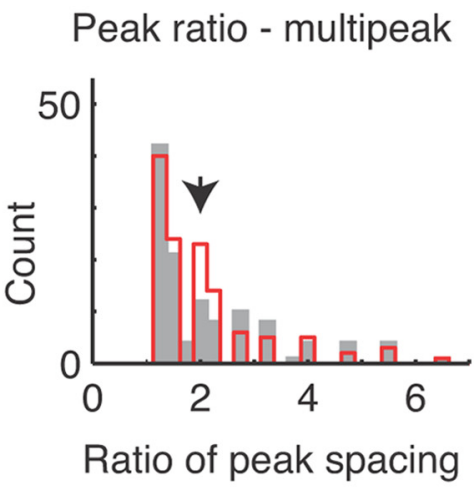

Figure 6. BF variability is due to spatial variability. $A$, Scatter plot of median BF of all responsive neurons in each paired imaging plane. The median BF is similar for each pair of imaging fields. $\boldsymbol{B}$, Percentage of single-peaked neurons in each paired imaging field. Horizontal bars indicate the mean for each group. Mean values in each group were similar (L2/3, $59 \pm 7 \%$ SEM; L4, $65 \pm 7 \%$ SEM; $p>0.3$, paired $t$ test). C, Cumulative distribution functions displaying a measure of frequency-tuning curve bandwidth (BW $\left.{ }_{75}\right)$ for single-peaked L2/3 (black) and L4 (red; median $_{\mathrm{L} 2 / 3}, 0.23 \pm 0.18$ IQR octaves; median $_{\mathrm{L} 4}, 0.28 \pm 0.18$ IQR octaves; $p<0.001$, Wilcoxon rank sum). D, BF variability $\left(I_{\mathrm{Q}} \mathrm{R}_{\mathrm{BF}}\right)$ for only single-peak neurons in paired imaging fields. Each set of connected dots represents the $\mathrm{IQR} \mathrm{R}_{\mathrm{BF}}$ for all responding neurons within a pair of imaging fields. Horizontal bars indicate the medians of each group

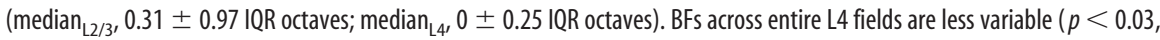
Wilcoxon sign rank). $E$, Cumulative distribution functions displaying local ( $<100 \mu \mathrm{m}$ radius) BF variability of only single-peak neurons in L2/3 (black) or L4 (red). Local BF variability is less in L4 (median ${ }_{\mathrm{L} 2 / 3}, 0.25 \pm 0.875$ IQR octaves; median $_{\mathrm{L} 4}, 0 \pm 0.375 \mathrm{IQR}$ octaves; $p<10^{-8}$, Wilcoxon rank sum). $\boldsymbol{F}$, Distributions of ratios of peak frequencies of multipeak neurons in L2/3 (gray) and L4 (red) are similar. Arrow indicates secondary peak representing octave spacing.

exhibited a higher level of $\mathrm{IQR}_{\mathrm{BF}}$ than single-peak $\mathrm{L} 4$ neurons when analyzed either on a field-by-field basis (Fig. $6 D ; p<0.02$ ) or as a population (Fig. $6 E ; p<0.001$ ). In contrast, the $\mathrm{IQR}_{\mathrm{BF}}$ of multipeaked neurons was not significantly different across layers (median $\mathrm{IQR}_{\mathrm{BF}} \mathrm{L} 2 / 3,2.0 \pm 1.69 \mathrm{IQR}$ octaves; $\mathrm{L} 4,1.75 \pm 1.5 \mathrm{IQR}$ octaves; $p>0.4$, Wilcoxon rank sum test; not shown). The fact that single-peak L2/3 neurons continued to exhibit higher levels of heterogeneity than L4 neurons reaffirms that the higher BF heterogeneity in $\mathrm{L} 2 / 3$ is not due to misestimation of BF. Although the precise role of multipeaked neurons in auditory processing is largely speculative at this time, it has been postulated that these neurons may be important for processing of complex sounds (Sutter and Schreiner, 1991; Kadia and Wang, 2003; Noreña et al., 2008). To investigate the spectral relationship between individual peaks in neurons with multipeak tuning curves in our dataset, we computed the ratio of all unique combinations of peaks in the tuning curve and compared across layers. We found similar spectral spacing (i.e., ratio between the peaks of multipeaked tuning curves; see Materials and Methods) in both $\mathrm{L} 2 / 3$ and $\mathrm{L} 4(\mathrm{~L} 2 / 3,1.68 \pm 1.41 \mathrm{IQR}$; L4, $1.68 \pm 0.96$ IQR). Notably for both L2/3 and L4, we observed a prominent secondary peak near 2 , which corresponds to octave spacing between peaks within a single tuning curve (Fig. 6F, arrow). A median value for both distributions (1.68 or 0.75 octaves) is consistent with previous reports on multipeaked neurons in cat and monkey and corresponds roughly to second and third harmonics in a harmonic complex (Sutter and Schreiner, 1991; Kadia and Wang, 2003; Noreña et al., 2008).

Collectively, these results demonstrate the existence of local scale response heterogeneity in the L2/3 compared to L4 and that increased BF heterogeneity in L2/3 cannot solely be attributable to the multipeaked nature of frequency-tuning curves.

\section{Differences in levels of correlated activity between $\mathrm{L} 2 / 3$ and $\mathrm{L} 4$ suggest different circuitry}

The homogeneous local organization in L4 suggests that a common stimulus-related input to these neurons exists (i.e., feedforward from the thalamus), whereas the heterogeneous organization in L2/3 predicts a more diverse range of inputs to these neurons (i.e., connectivity from either diverse populations of thalamorecipient neurons or widespread intralaminar connections or input from nonlemniscal thalamic nuclei). These potential differences in cortical connectivity (common, feedforward input vs diverse, lateral connections) can be inferred from measuring correlated activity within groups of neurons. Thus, to understand how sensory information is represented in populations of neurons in the different layers, we characterized the responses of groups of neurons in the different layers by computing signal (response similarity) and noise correlations (trial-to-trial response fluctuations) between simultaneously imaged neurons.

A smooth spatial gradient of BF implies that neighboring neurons are driven by topographically organized inputs representing 
nearby frequencies. In this scenario, neighboring neurons would be expected to show a high level of stimulus-related correlated activity (signal correlations). Indeed, in the imaged fields shown in Figure 3 , we found significant signal correlations between pairs of neurons in each imaging plane (both $p<10^{-21}$, paired $t$ test comparing actual data with shuffled version of the data; Fig. $7 A, B)$. When compared across imaging planes, signal correlations were larger in L4 (mean $\mathrm{SC}_{\mathrm{L} 2 / 3}, 0.25$; mean $\mathrm{SC}_{\mathrm{L} 4}, 0.37 ; p<10^{-11}$, unpaired $t$ test; Fig. 7C). Moreover, when we used a correction method to remove the contribution of noise correlations to signal correlations (SC-NC), the values were roughly similar (Fig. $7 C$, inset). In other paired imaging sites, we tended to find similar outcomes ( 11 of 16 cases; Fig. $8 A-C)$. Across the population, we found significantly higher signal correlations in L4 compared with $\mathrm{L} 2 / 3$ (median $\mathrm{SC}_{\mathrm{L} 2 / 3}$, $0.39 \pm 0.31$; median $\mathrm{SC}_{\mathrm{L} 4}, 0.48 \pm 0.41$; $p<10^{-19}$, Wilcoxon rank sum; median $\mathrm{SC} \mathrm{NC}_{\mathrm{L} 2 / 3}, 0.25 \pm 0.30$; median SC$\mathrm{NC}_{\mathrm{L} 4}, 0.49 \pm 0.32 ; p<10^{-26}$, Wilcoxon rank sum; Fig. $9 A-D$ ).

While signal correlations reflect common (shared) input into groups of neurons that is stimulus related, pairwise trial-to-trial response covariance (i.e., noise correlation) is stimulus independent and likely reflects the state of the cortical network during stimulus presentation (Kohn and Smith, 2005) due to functional connectivity or shared, stimulus-independent input. Consistent with prior results (Rothschild et al., 2010), we found significant noise correlations between pairs of neurons in both imaging planes (both $p<10^{-24}$, paired $t$ test comparing actual data with shuffled version of data; Fig. $7 D--F)$. When compared across imaging planes, noise correlations were smaller in $\mathrm{L} 4$ than in $\mathrm{L} 2 / 3$ for this pair of imaging fields (median $\mathrm{NC}_{\mathrm{L} 2 / 3}$, 0.26 ; median $\mathrm{NC}_{\mathrm{L} 4}, 0.09 ; p<10^{-30}$, unpaired $t$ test; Fig. $7 F$ ). As observed with signal correlations, we found few cases in which the average noise correlation value for all neuron pairs in the field of view was the same as or higher than the noise correlation for the corresponding L4 imaging location (Fig. 8D-F). Across the population of L2/3 and L4, we found significantly higher noise correlations in $\mathrm{L} 2 / 3$ compared with $\mathrm{L} 4$ (median $\mathrm{NC}_{\mathrm{L} 2 / 3}, 0.22$; median $\mathrm{NC}_{\mathrm{L} 4}, 0.20 ; p<0.01$, Wilcoxon rank sum; Fig. 9D-F).

In both populations, signal and noise correlations were positively correlated, indicating that when two neurons had similar stimulus-driven response properties (i.e., high SC), their responses also tended to covary with respect to their mean response on a trial-by-trial basis (Fig. $10 \mathrm{~A}$ ), implying that similarly tuned neurons might be connected (Ko et al., 2011).

Together, these results suggest that $\mathrm{L} 4$ neurons receive a larger fraction of shared feed-forward stimulus-dependent inputs and that L2/3 neurons show a higher level of stimulus-independent interconnectivity, which is consistent with the serial nature of
B

4

C
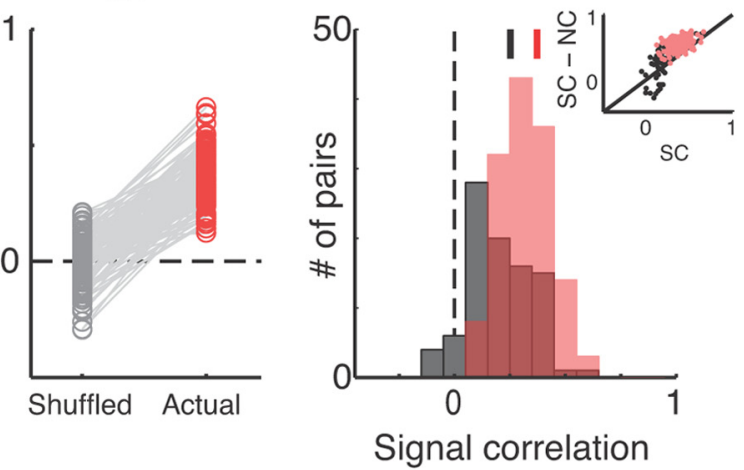

F

L4
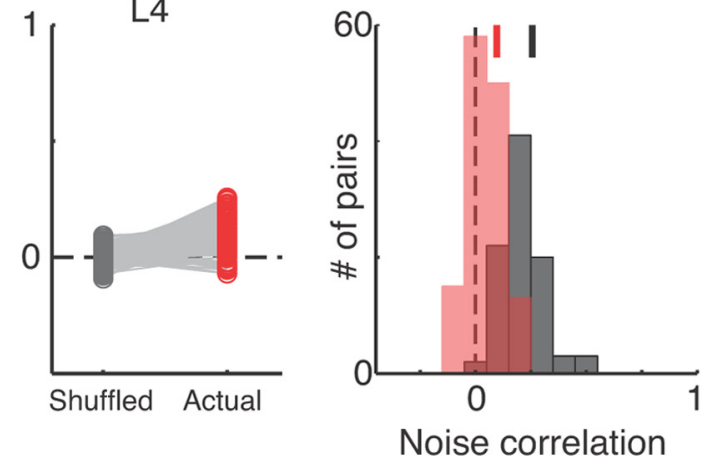

Figure 7. Signal and noise correlations between neurons in $L 2 / 3$ and $L 4$ are different. $A$, Significant pairwise signal crosscorrelations in L2/3 for an example imaging field. Light gray circles show correlations obtained from a randomized version of the data (see Materials and Methods; $n=91$ pairs; $p=10^{-24}$, paired $t$ test). $\boldsymbol{B}$, Significant pairwise signal cross-correlations in L4 $\left(n=136\right.$ pairs; $p=10^{-5}$, paired $t$ test). C, Comparison of distributions of signal correlation values in L2/3 (black) and L4 (red). values. Each symbol represents an SC value ( $x$-axis) or SC values corrected for the contributions of noise correlations ( $y$-axis) for a pair of neurons in L2/3 (black) or L4 (red). $\boldsymbol{D}, \boldsymbol{E}$, Significant pairwise noise correlations in L2/3 and $L 4$ in an example field ( $p=$ $10^{-39}$ and $p=10^{-23}$, respectively). $\boldsymbol{F}$, Comparison of distributions of noise correlation values in L2/3 (black) and L4 (red). Vertical lines indicate means of distributions $\left(p<10^{-30}\right.$, unpaired $t$ test).

cortical processing and extensive intracortical connections in $\mathrm{L} 2 / 3$.

In vitro studies have shown that connection probabilities between pairs of neurons in A1 decrease with increasing distance from each other (Levy and Reyes, 2012). Furthermore, neurons that are spatially distant from each other are expected to share fewer inputs. Thus, it is expected that both signal and noise correlations decrease with distance. The spatial properties of this decrease are typically used as a measure of likelihood of connectivity, which is believed to influence cortical receptive field properties like frequency tuning and spectral integration (Schreiner et al., 2000; Oswald et al., 2006). In our data, cell density was similar in both imaging planes (L2/3 and L4; Fig. 10B). To evaluate the spatial extent of correlated activity in A1 in vivo, we plotted signal and noise correlation values for pairs of neurons as a function of the spatial separation of the pair (Fig. 10C,D). For this, we grouped the correlation values according to spatial separation of the neuron pairs ( $10 \mu \mathrm{m}$ increments starting at $15 \mu \mathrm{m}$ ) and calculated the median values in each distance bin. Because the likelihood of finding a cell pair separated by more than $\sim 120 \mu \mathrm{m}$ was extremely low (Fig. 10B), we limited our graphical representation to this upper bound. As expected, signal and noise correlations for both groups decreased with distance (Fig. 10C,D), indicating that nearby pairs of neurons in vivo are more likely to have similar 
A

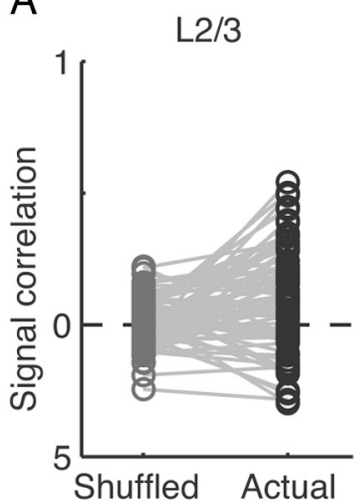

B
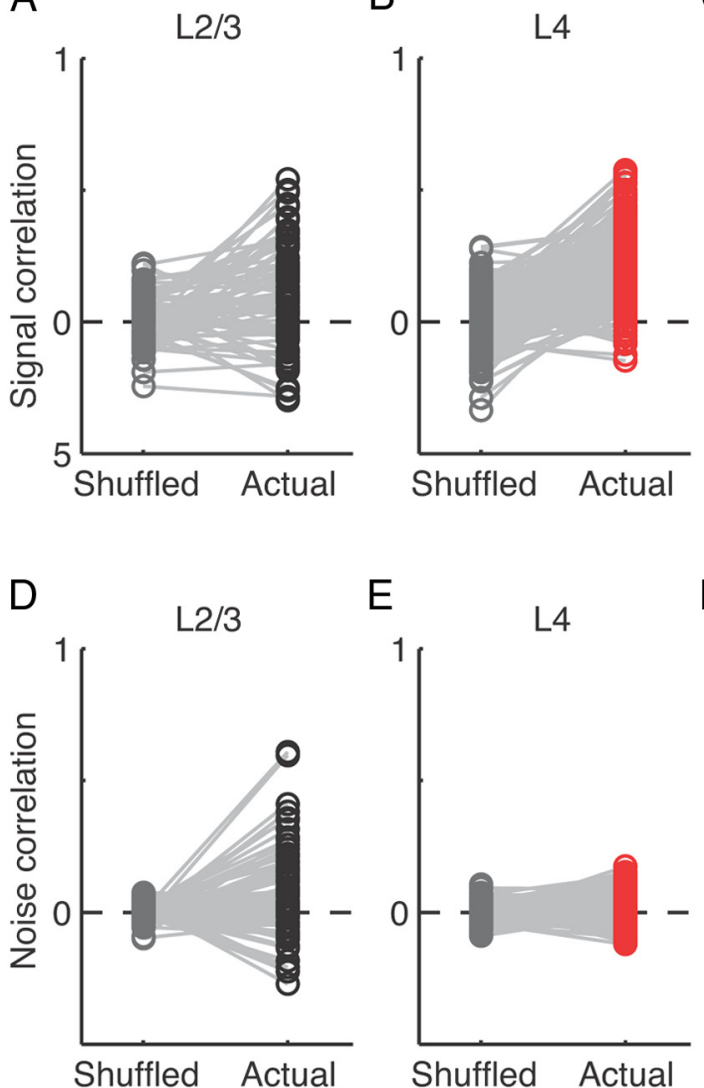

E

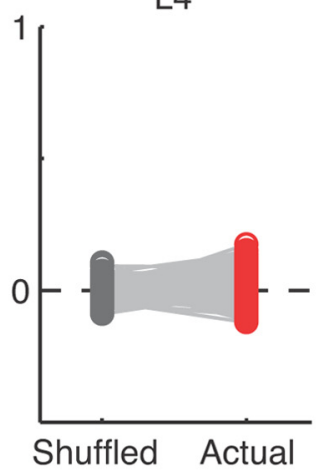

C

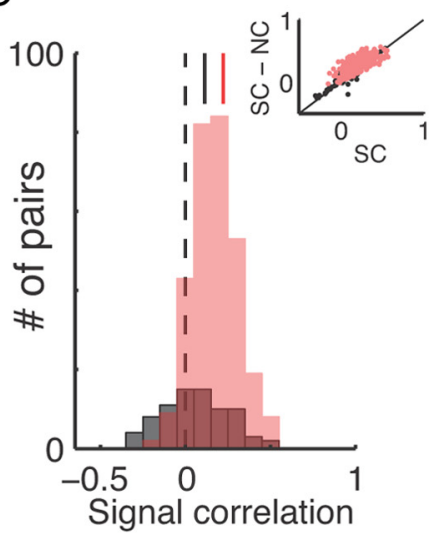

Just as previous imaging studies of superficial (L2/3) mouse A1 reported heterogeneous frequency organization on local $(<100 \mu \mathrm{m})$ scales, recent investigation of the frequency representation in the mouse using lower spatial resolution sampling methods (i.e., in vitro voltage-sensitive dye-imaging and traditional electrophysiological methods) also suggested that the spatial organization in L2/3 might be less precise than in L4 (Hackett et al., 2011; Guo et al., 2012). For example, in vitro voltage-sensitive dye-imaging studies which report voltage changes across all neuronal compartments showed that while loci of L4 activity due to stimulation of ventral division of medial geniculate body (vMGB) shifted systematically with the vMGB stimulation location, loci of activity in L2/3 due to the same stimulation shifted weakly and did not reach significance (Hackett et al., 2011). In addition, using traditional extracellular recording methods to map the frequency representation in A1, Guo et al. (2012) stated that tonotopy is represented equivalently in deep, middle, and superficial layers of the cortex, even though pooling recording sites with regular and irregular tuning profiles (i.e., presumably all responsive neurons) can create the appearance of substantially degraded tonotopy outside of thalamic input layers. Together, these two reports suggest that the representation of sound frequency may be transformed between L4 and L2/3. Indeed, in our data, we directly demonstrate differences in the frequency representation in populations of individual neurons in L4 and L2/3. However, disparities between results obtained with electrophysiological and single cell imaging approaches still remain. In our experiments, while we observed that within each animal

stimulus-driven responses than distant pairs. The distancedependent decrease in signal correlations was less in L4 compared to L2/3 (Fig. 10C), suggesting a more homogeneous stimulusdependent input across the L4 imaging fields. Similarly, the distance dependent decrease in noise correlation was also less in L4 when compared to L2/3 (Fig. 10C). However, the overall values for L4 were lower than the values in L2/3 up to $\sim 70 \mu \mathrm{m}$ (Fig. 10D), leading to an overall lower median value for L4 noise correlation that was observed for the population (Fig. $9 F$ ). In total, this analysis provides a spatial profile of correlated activity within A1 in vivo, which is consistent with the known organization of cortical circuits and extensive intracortical connections in $\mathrm{L} 2 / 3$.

\section{Discussion}

Our results reveal a more homogeneous spatial organization in L4 when directly compared to L2/3, and also show higher frequency selectivity in L2/3 compared to L4. Moreover, our findings bridge the seemingly differing results obtained with more traditional methodological approaches and show directly that a transformation of the frequency representation occurs between L4 and L2/3 within A1. the average $\mathrm{BF}$ in each paired imaging field (i.e., L2/3 and L4) matched (Fig. $6 A$ ), local heterogeneity emerges in L2/3, suggesting that it is created in L2/3 on top of an overall backbone of a large-scale tonotopic organization. Furthermore, when we limited our analysis to neurons with single-peaked tuning, as done by Guo et al. (2012), we continued to observe a significant amount of BF variability in $\mathrm{L} 2 / 3$ on local $(<100 \mu \mathrm{m})$ scales compared to the BF variability of L4 neurons (Fig. $6 D, E$ ). This organizational feature of $\mathrm{L} 2 / 3$ neurons (i.e., heterogeneity of tuning) was also observed by Rothschild et al. (2010) when they limited their analysis to neurons with nonpatchy frequency response areas (presumably, single-peaked neurons). Why might the two methodological approaches arrive at such seemingly different conclusions? One explanation might be because voltage-sensitive dyes and electrophysiological studies sample at much lower spatial densities than two-photon imaging studies are capable of, and thus might not uncover local differences. A second, alternative explanation could be that due to inherent limitations of electrophysiological recordings such as intrinsic electrode bias (Towe and Harding, 1970; Stone, 1973; Levick and Cleland, 1974; Humphrey and Corrie, 1978; Sommer and Wurtz, 2000), one might unintentionally oversample selected subpopulations of A1 neurons 
and perhaps ignore small neurons, poorly tuned neurons, or neurons with low baseline firing rates (Hromádka et al., 2008; Oviedo et al., 2010). Third, insufficient isolation of single units may average over multiple neurons and thus might miss neuron-by-neuron differences. Our imaging study suggests that individual neuron-by-neuron differences might be more prevalent in L2/3, particularly on local $(<100 \mu \mathrm{m})$ scales, and thus the effects of electrophysiological sampling densities and sampling bias might be stronger in $\mathrm{L} 2 / 3$ and can be overcome by high-resolution optical imaging approaches.

Finally, what is important to note here is that both previous imaging studies detected tonotopic gradients in $\mathrm{L} 2 / 3$ on large spatial scales demonstrating that tonotopy is, and continues to be, an organizational feature of $\mathrm{A} 1$, even in $\mathrm{L} 2 / 3$ (Bandyopadhyay et al., 2010; Rothschild et al., 2010). However, where imaging and traditional electrophysiology findings diverge is in the reported spatial precision of tonotopic maps in L2/3. Electrophysiological studies observe tonotopy, but emphasize the large $(\sim 100 \mu \mathrm{m}$; based on Guo et al., 2012, their Fig. 1) spatial scale precision of the maps and the response homogeneity among sampled neurons. In contrast, imaging studies, including the current one, observe tonotopy in A1 (Bandyopadhyay et al., 2010; Rothschild et al., 2010), but emphasize the differences on local $(<100 \mu \mathrm{m})$ spatial scales. This is primarily because the high spatial resolution of imaging techniques allows for the probing of the fine spatial organization of cortical circuits in a manner that extracellular electrodes simply are unable to achieve. Thus, imaging studies can reveal the differences of the spatial organization among local populations of single neurons within $\sim 100 \mu \mathrm{m}^{2}$ regions and, in the current study, compare across layers. In particular, the median $\mathrm{BF}$ in $\sim 100 \mu \mathrm{m}^{2}$ regions is similar between paired imaging fields, indicating that a tonotopic structure is present across layers (Fig. 6A), but other measures of organization differ between them, suggesting that a new local organizational pattern may emerge in L2/3. Thus, imaging and electrophysiological studies offer complementary perspectives on the local organization of A1.

Our data suggest that increased heterogeneity of frequency preference in L2/3 neurons is likely due to a widespread sampling of inputs from L4 with different BFs by L2/3 (Bandyopadhyay et al., 2010; Chen et al., 2011; Hackett et al., 2011). This widespread sampling of inputs to $\mathrm{L} 2 / 3$ could, in principle, lead to a steeper decline in correlated activity in L2/3 compared to L4 (Fig. 10), and thus serve as the basis for an emergent organizational feature within L2/3. Furthermore, in our study, the similar fractions of
B
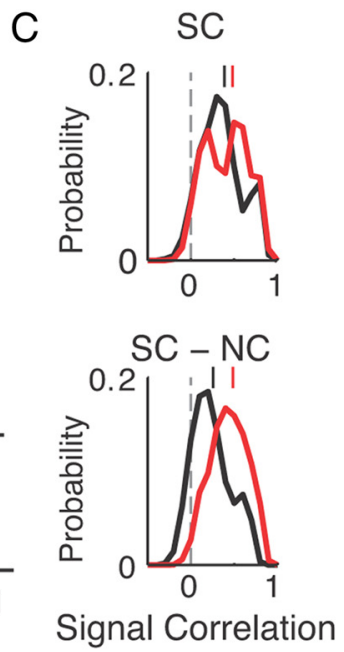

D L2/3

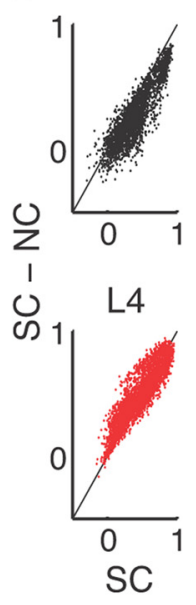

$\mathrm{F}$

G

L4
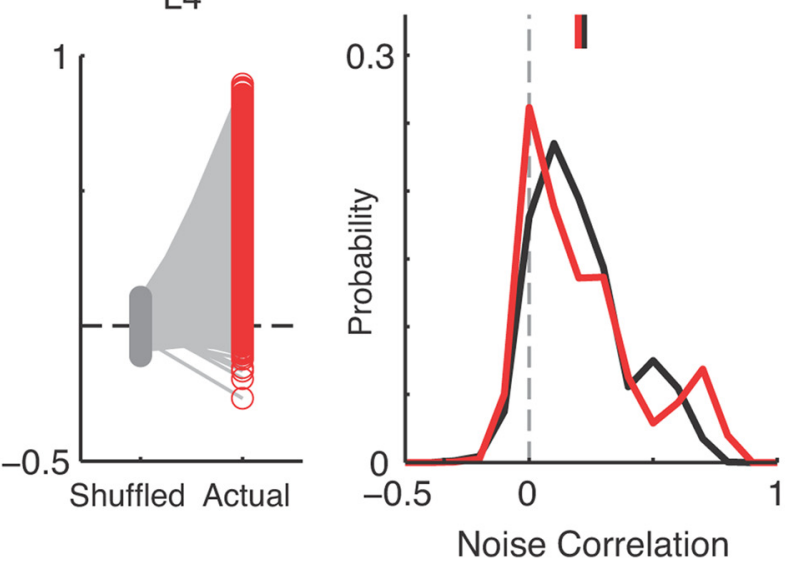

Figure 9. Differences in signal and noise correlations in $L 2 / 3$ and $L 4$ neuron populations. $\boldsymbol{A}, \boldsymbol{B}$, Significant pairwise signal cross-correlations (SC) in the populations of L2/3 (black) and L4 (red). Light gray circles represent correlations obtained from a randomized version of the data; black or red circles indicate correlation values from the actual data. $C$, Distributions of $S C$ values values corrected for the contribution of noise correlations. Vertical lines indicate medians of distributions. $p<10^{-9}$ for SC; $p<$ $10^{-26}$ for SC-NC (Wilcoxon rank sum test). D, Scatter plots comparing SC values and SC-NC values. Each symbol represents SC value $\boldsymbol{E}, \boldsymbol{F}$, Significant pairwise noise correlations in L2/3 and L4 layers. Light gray circles represent correlations obtained from a randomized version of the data; black or red circles indicate correlation values from the actual data. $\mathbf{G}$, Comparison of distributions of noise correlation values in L2/3 (black) and L4 (red). Vertical lines indicate medians of distributions ( $p=0.009$, Wilcoxon rank sum).

multipeaked neurons in both layers also suggests that frequency heterogeneity in $\mathrm{L} 2 / 3$ is not predominantly due to presence of multipeaked neurons.

The high levels of response heterogeneity in mouse L2/3 may be due to smaller brain size in mice, as high levels of response heterogeneity also exist in L2/3 of mouse visual cortex (Smith and Hausser, 2010; Bonin et al., 2011) as well as rodent somatosensory cortex (Brumberg et al., 1999; Sato et al., 2007). However, since topographic maps such as orientation maps in visual cortex that are present in carnivores are absent in rodents, including large rodents such as squirrels (Van Hooser et al., 2003), brain size might only play a limited role, and substantial laminar transformations and high heterogeneity might be a general feature of sensory processing, at least in rodents. Moreover, our results are consistent with in vivo two-photon $\mathrm{Ca}^{2+}$ imaging experiments using a high- and low-affinity $\mathrm{Ca}^{2+}$ indicator, suggesting that 
A

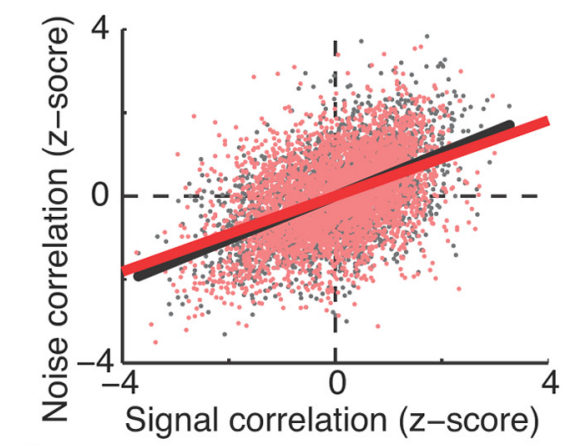

C

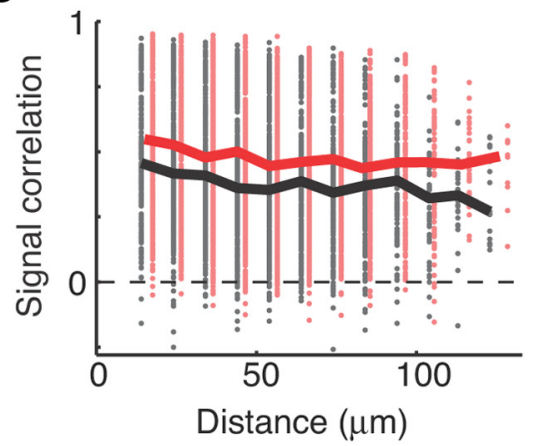

B

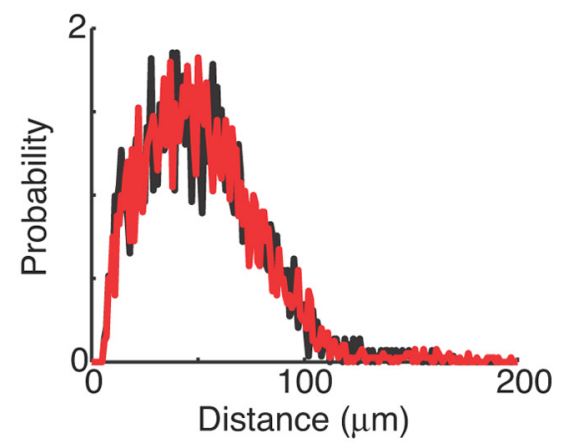

D

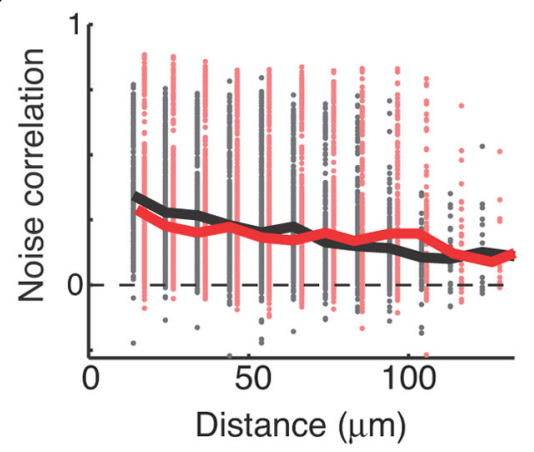

Figure 10. Signal and noise correlations are correlated and decrease with distance. $\boldsymbol{A}$, Noise and signal correlations for individual cell pairs are correlated. Data were normalized for comparison across animals and imaged regions by computing $z$ scores (see Materials and Methods; slope $\mathrm{L} 2 / 3_{3}, 0.52$; slope $\left._{\mathrm{L} 4}, 0.45 ; p<0.002\right)$. $\boldsymbol{B}$, Display of cell densities in the two imaging planes (L2/3, black; $L 4$, red). C, Signal correlations in L2/3 and L4 as function of cell-pair distance. Data were grouped into $\sim 10 \mu \mathrm{m}$ bins, and all correlations values occurring within that distance increment were plotted. Data from the two groups (L2/3 and L4) were offset for clarity. Lines (black and red) represent the median values in each distance bin. D, Conventions are as in C. Noise correlations in L2/3 and $L 4$ are shown as function of cell-pair distance.

sensory-evoked response properties of inputs to L2/3 (i.e., L4) are more homogeneous than their spiking outputs (Bandyopadhyay et al., 2010). This implies that the increased heterogeneity in L2/3 may be an emergent property of L2/3, an idea put forth for other sensory systems (Brumberg et al., 1999). Moreover, given that this feature of heterogeneous and spatially intermingled organization in L2/3 neurons has been observed in other rodent sensory systems using either extracellular recording methods or imaging approaches (Brumberg et al., 1999; Sato et al., 2007; Bonin et al., 2011), it is likely to represent a general feature of sensory cortical organization, at least in rodents.

The significant differences in response properties and frequency representation across the earliest laminar stages of A1 suggest that sound frequency is not the only stimulus feature represented by L2/3. For example, complex auditory stimuli, like vocalizations, often contain sound energy in nonneighboring frequency bands. It is possible that the organization within the supragranular layer of $\mathrm{A} 1$ is specialized for the extraction and representation of such stimulus features, and therefore may be critical for processing such behaviorally relevant stimuli. Finally, the differences between layers that we have described in A1 provide direct evidence of a transformation of sensory representation across cortical layers and might represent a general feature of sensory cortical circuits.

\section{References}

Abeles M, Goldstein MH Jr (1970) Functional architecture in cat primary auditory cortex: columnar organization and organization according to depth. J Neurophysiol 33:172-187. Medline
Armstrong-James M, Fox K, Das-Gupta A (1992) Flow of excitation within rat barrel cortex on striking a single vibrissa. J Neurophysiol 68:1345-1358. Medline

Atencio CA, Schreiner CE (2010) Laminar diversity of dynamic sound processing in cat primary auditory cortex. J Neurophysiol 103: 192-205. CrossRef Medline

Atencio CA, Sharpee TO, Schreiner CE (2009) Hierarchical computation in the canonical auditory cortical circuit. Proc Natl Acad Sci U S A 106:21894-21899. CrossRef Medline

Averbeck BB, Latham PE, Pouget A (2006) Neural correlations, population coding and computation. Nat Rev Neurosci 7:358-366. CrossRef Medline

Bandyopadhyay S, Shamma SA, Kanold PO (2010) Dichotomy of functional organization in the mouse auditory cortex. Nat Neurosci 13:361-368. CrossRef Medline

Bonin V, Histed MH, Yurgenson S, Reid RC (2011) Local diversity and fine-scale organization of receptive fields in mouse visual cortex. J Neurosci 31:18506-18521. CrossRef Medline

Brumberg JC, Pinto DJ, Simons DJ (1999) Cortical columnar processing in the rat whiskerto-barrel system. J Neurophysiol 82:18081817. Medline

Chen X, Leischner U, Rochefort NL, Nelken I, Konnerth A (2011) Functional mapping of single spines in cortical neurons in vivo. $\mathrm{Na}$ ture 475:501-505. CrossRef Medline

Cohen MR, Kohn A (2011) Measuring and interpreting neuronal correlations. Nat Neurosci 14:811-819. CrossRef Medline

Guo W, Chambers AR, Darrow KN, Hancock KE, Shinn-Cunningham BG, Polley DB (2012) Robustness of cortical topography across fields, laminae, anesthetic states, and neurophysiological signal types. J Neurosci 32: 9159-9172. CrossRef Medline

Hackett TA, Barkat TR, O’Brien BM, Hensch TK, Polley DB (2011) Linking topography to tonotopy in the mouse auditory thalamocortical circuit. J Neurosci 31:2983-2995. CrossRef Medline

Hromádka T, Deweese MR, Zador AM (2008) Sparse representation of sounds in the unanesthetized auditory cortex. PLoS Biol 6:e16. CrossRef Medline

Hubel DH, Wiesel TN (1977) Ferrier lecture. Functional architecture of macaque monkey visual cortex. Proc R Soc Lond B Biol Sci 198:1-59. CrossRef Medline

Humphrey DR, Corrie WS (1978) Properties of pyramidal tract neuron system within a functionally defined subregion of primate motor cortex. J Neurophysiol 41:216-243. Medline

Josić K, Shea-Brown E, Doiron B, de la Rocha J (2009) Stimulus-dependent correlations and population codes. Neural Comput 21:2774-2804. CrossRef Medline

Kaas JH (2011) The evolution of auditory cortex: the core areas. In: The auditory cortex (Winer JA, Schreiner CE, eds), pp 407-427. New York: Springer.

Kadia SC, Wang X (2003) Spectral integration in Al of awake primates: neurons with single- and multipeaked tuning characteristics. J Neurophysiol 89:1603-1622. Medline

Ko H, Hofer SB, Pichler B, Buchanan KA, Sjöström PJ, Mrsic-Flogel TD (2011) Functional specificity of local synaptic connections in neocortical networks. Nature 473:87-91. CrossRef Medline

Kohn A, Smith MA (2005) Stimulus dependence of neuronal correlation in primary visual cortex of the macaque. J Neurosci 25:3661-3673. CrossRef Medline

Levick WR, Cleland BG (1974) Selectivity of microelectrodes in recordings from cat retinal ganglion cells. J Neurophysiol 37:1387-1393. Medline

Levy RB, Reyes AD (2012) Spatial profile of excitatory and inhibitory syn- 
aptic connectivity in mouse primary auditory cortex. J Neurosci 32:56095619. CrossRef Medline

Madisen L, Zwingman TA, Sunkin SM, Oh SW, Zariwala HA, Gu H, Ng LL, Palmiter RD, Hawrylycz MJ, Jones AR, Lein ES, Zeng H (2010) A robust and high-throughput Cre reporting and characterization system for the whole mouse brain. Nat Neurosci 13:133-140. CrossRef Medline

Mountcastle VB (1997) The columnar organization of the neocortex. Brain 120:701-722. CrossRef Medline

Nimmerjahn A, Kirchhoff F, Kerr JN, Helmchen F (2004) Sulforhodamine 101 as a specific marker of astroglia in the neocortex in vivo. Nat Methods 1:31-37. CrossRef Medline

Noreña AJ, Gourévitch B, Pienkowski M, Shaw G, Eggermont JJ (2008) Increasing spectrotemporal sound density reveals an octave-based organization in cat primary auditory cortex. J Neurosci 28:8885-8896. CrossRef Medline

Oswald AM, Schiff ML, Reyes AD (2006) Synaptic mechanisms underlying auditory processing. Curr Opin Neurobiol 16:371-376. CrossRef Medline

Oviedo HV, Bureau I, Svoboda K, Zador AM (2010) The functional asymmetry of auditory cortex is reflected in the organization of local cortical circuits. Nat Neurosci 13:1413-1420. CrossRef Medline

Rothschild G, Nelken I, Mizrahi A (2010) Functional organization and population dynamics in the mouse primary auditory cortex. Nat Neurosci 13:353-360. CrossRef Medline

Sakata S, Harris KD (2009) Laminar structure of spontaneous and sensoryevoked population activity in auditory cortex. Neuron 64:404-418. CrossRef Medline

Sato TR, Gray NW, Mainen ZF, Svoboda K (2007) The functional microarchitecture of the mouse barrel cortex. PLoS Biol 5:e189. CrossRef Medline

Schreiner CE, Winer JA (2007) Auditory cortex mapmaking: principles, projections, and plasticity. Neuron 56:356-365. CrossRef Medline
Schreiner CE, Read HL, Sutter ML (2000) Modular organization of frequency integration in primary auditory cortex. Annu Rev Neurosci 23:501-529. CrossRef Medline

Sharpee TO, Atencio CA, Schreiner CE (2011) Hierarchical representations in the auditory cortex. Curr Opin Neurobiol 21:761-767. CrossRef Medline

Smith SL, Häusser M (2010) Parallel processing of visual space by neighboring neurons in mouse visual cortex. Nature neuroscience 13:1144-1149. CrossRef Medline

Sommer MA, Wurtz RH (2000) Composition and topographic organization of signals sent from the frontal eye field to the superior colliculus. J Neurophysiol 83:1979-2001. Medline

Stiebler I, Neulist R, Fichtel I, Ehret G (1997) The auditory cortex of the house mouse: left-right differences, tonotopic organization and quantitative analysis of frequency representation. J Comp Physiol A Neuroethol Sens Neural Behav Physiol 181:559-571. CrossRef

Stone J (1973) Sampling properties of microelectrodes assessed in the cat's retina. J Neurophysiol 36:1071-1079. Medline

Sutter ML, Schreiner CE (1991) Physiology and topography of neurons with multipeaked tuning curves in cat primary auditory cortex. J Neurophysiol 65:1207-1226. Medline

Towe AL, Harding GW (1970) Extracellular microelectrode sampling bias. Exp Neurol 29:366-381. CrossRef Medline

Van Hooser SD, Heimel JA, Nelson SB (2003) Receptive field properties and laminar organization of lateral geniculate nucleus in the gray squirrel (Sciurus carolinensis). J Neurophysiol 90:3398-3418. CrossRef Medline

Viswanathan S, Bandyopadhyay S, Kao JP, Kanold PO (2012) Changing microcircuits in the subplate of the developing cortex. J Neurosci 32: 1589-1601. CrossRef Medline 